\title{
An optimal symbolic calculus on Besov algebras
}

\author{
Gérard Bourdaud $^{\mathrm{a}, *}$, Madani Moussai $^{\mathrm{b}}$, Winfried Sickel ${ }^{\mathrm{c}}$ \\ a Institut de Mathématiques de Jussieu, Projet d'analyse fonctionnelle, Case 186, 4, place Jussieu, 75252 Paris Cedex 05, France \\ ${ }^{\mathrm{b}}$ Department of Mathematics, LMPA, University of M'Sila, P.O. Box 166, 28000 M'Sila, Algeria \\ c Mathematisches Institut, FSU Jena, Ernst-Abbe-Platz 1-2, O7743 Jena, Germany
}

Received 9 January 2006; received in revised form 4 April 2006; accepted 9 June 2006

Available online 25 September 2006

\begin{abstract}
In this paper we consider Besov algebras on $\mathbb{R}$, that is Besov spaces $B_{p, q}^{s}(\mathbb{R})$ for $s>1 / p$. For $s>1+(1 / p), p>4 / 3$, and $q \geqslant p$ we prove that the above algebras have a maximal symbolic calculus in the following sense: for any function $f$ belonging locally to $B_{p, q}^{s}(\mathbb{R})$ and such that $f(0)=0$, the associated superposition operator $T_{f}(g):=f \circ g$ takes $B_{p, q}^{s}(\mathbb{R})$ to itself. (C) 2006 L'Association Publications de l'Institut Henri Poincaré. Published by Elsevier B.V. All rights reserved
\end{abstract}

\section{Résumé}

On considère les algèbres de Besov sur la droite réelle, autrement dit les espaces de Besov $B_{p, q}^{s}(\mathbb{R})$ pour $s>1 / p$. Sous les hypothèses $s>1+(1 / p), p>4 / 3$ et $q \geqslant p$, on établit que ces algèbres possèdent un calcul symbolique maximal au sens suivant : pour toute fonction $f$ appartenant localement à $B_{p, q}^{s}(\mathbb{R})$ et telle que $f(0)=0$, on a $f \circ g \in B_{p, q}^{s}(\mathbb{R})$ pour tout $g \in B_{p, q}^{s}(\mathbb{R})$. ๔ 2006 L'Association Publications de l'Institut Henri Poincaré. Published by Elsevier B.V. All rights reserved

MSC: 46J10; 46E35; 47H30

Keywords: Besov spaces; Commutative Banach algebra; Symbolic calculus; Superposition operators

\section{Introduction}

Let us denote by $C_{b}(\mathbb{R})$ the Banach algebra of bounded continuous functions in $\mathbb{R}$. Assume that $E$ is a subalgebra of $C_{b}(\mathbb{R})$. Assume further that $E$ is endowed with a norm which renders the canonical injection $E \hookrightarrow C_{b}(\mathbb{R})$ continuous, and such that $E$ is a Banach algebra. In such an algebra, the symbolic calculus consists in associating to any function $g \in E$ the composed function $f \circ g$, under some appropriate conditions on $f$. Thus we say that a function $f$, defined on a subset $\Omega$ of $\mathbb{C}$, operates in $E$ if we have $f \circ g \in E$ for any $g \in E$ whose range is contained in $\Omega$. The above notion makes sense in any function space $E$, even if it is not a Banach algebra. The operator $T_{f}: g \mapsto f \circ g$ is often referred as a superposition operator.

\footnotetext{
* Corresponding author.

E-mail addresses: bourdaud@ccr.jussieu.fr (G. Bourdaud), mmoussai@yahoo.fr (M. Moussai), sickel@ minet.uni-jena.de (W. Sickel).
} 
It is well known that any holomorphic function $f$, defined on an open subset of $\mathbb{C}$ - and satisfying $f(0)=0$ in case $1 \notin E-$ operates in $E$. The same property holds for a real analytic function, under certain assumptions on $E$. We refer to [11, Chapter VIII, 3.9 and 8.1$]$ for more details.

In case $\mathcal{D}(\mathbb{R}) \subset E$ it is easily seen that any function $f: \mathbb{R} \rightarrow \mathbb{C}$ which operates in $E$ belongs necessarily to $E_{\text {loc }}$.

We shall speak of a maximal symbolic calculus if any function $f \in E_{\mathrm{loc}}-$ satisfying $f(0)=0$ in case $1 \notin E-$ operates in $E$, and of a minimal symbolic calculus if the only operating functions are the analytic ones.

According to a celebrated theorem of Kahane and Katznelson (see [11, Chapter VIII, 8.6]), the algebra $A(\mathbb{R})$ of the Fourier transforms of integrable functions has a minimal symbolic calculus. On the contrary, the algebra $C_{b}(\mathbb{R})$ itself has a maximal symbolic calculus; the same holds for the classical Sobolev algebras $W_{p}^{m}(\mathbb{R}), m$ integer $\geqslant 2$, $1 \leqslant p \leqslant \infty$, see [2].

Here we deal with Besov spaces. For the precise definition of these spaces we refer to $[1,13,16]$. Throughout the paper we shall assume $p, q \in[1,+\infty]$, and $s>0$, unless otherwise stated.

Let us recall that $B_{p, q}^{s}(\mathbb{R})$ is a Banach algebra, embedded into $C_{b}(\mathbb{R})$, if $s>1 / p$, and that the following conditions are necessary for a function $f$ to operate in $B_{p, q}^{s}(\mathbb{R})$ :

(A) $f \in B_{p, q}^{s}(\mathbb{R})_{\text {loc }}$,

(B) $f$ is locally Lipschitz continuous, cf. [3].

By a classical Sobolev embedding, we have (A) $\Rightarrow(\mathrm{B})$ if $s>1+(1 / p)$. Based on this remark, and the known results for the classical Sobolev spaces [2], we believe on the following conjecture.

Conjecture. In case $s>1+(1 / p)$ the Besov algebra $B_{p, q}^{s}(\mathbb{R})$ has a maximal symbolic calculus. More precisely, if $f: \mathbb{R} \rightarrow \mathbb{R}$ is any Borel function such that $f(0)=0$, then $f$ operates in $B_{p, q}^{s}(\mathbb{R})$ if and only if $f \in B_{p, q}^{s}(\mathbb{R})_{\text {loc. }}$.

Let us add a few comments to the case $0<s \leqslant 1+(1 / p)$. If $0<s<1$, then the following result is well known [3]: a Borel function $f$ such that $f(0)=0$ operates in $B_{p, q}^{s}(\mathbb{R})$ if and only if either $f$ is locally Lipschitz continuous (if $B_{p, q}^{s}(\mathbb{R}) \subset L_{\infty}(\mathbb{R})$ ) or $f$ is uniformly Lipschitz continuous (if $B_{p, q}^{s}(\mathbb{R}) \not \subset L_{\infty}(\mathbb{R})$ ). In case $1 \leqslant s \leqslant 1+(1 / p)$ the symbolic calculus turns out to be more mysterious. Indeed we expect that the conditions (A) and (B), together with $f(0)=0$, are not sufficient for $f$ to act on the space $B_{p, q}^{s}(\mathbb{R})$. A typical example is the Zygmund class $B_{\infty, \infty}^{1}(\mathbb{R})$, for which a full description of the symbolic calculus has been given in [6]. There a necessary and sufficient condition on $f$ was found which is stronger than these quoted conditions.

Notation. If $s$ is a real number, then $[s]$ denotes the integer part of $s$, i.e. the largest integer less than or equal to $s$. All functions are assumed to be real-valued. With $\|f\|_{p}$ we denote the $L_{p}$-norm on $\mathbb{R}$. As usual, constants $c, c_{1}, \ldots$ are strictly positive and depend only on the fixed parameters $s, p, q$, unless otherwise stated; their values may vary from line to line.

\section{The main theorem}

The following result represents a partial, but substantial, proof of the conjecture.

Theorem 1. Let us assume that the following three conditions hold:

- $s>1+(1 / p)$,

- $p>4 / 3$ or $1 / p<s-[s]$,

- $q \geqslant p$.

If $f: \mathbb{R} \rightarrow \mathbb{R}$ is a Borel function such that $f(0)=0$, then $f$ operates in $B_{p, q}^{s}(\mathbb{R})$ if and only if $f \in B_{p, q}^{s}(\mathbb{R})_{\text {loc. }}$

Some comments are in order. Theorem 1 remains valid under more general assumptions, e.g. also for

$$
1<p \leqslant \frac{4}{3} \quad \text { and } \quad s-[s] \notin\left[\frac{1}{p}-\frac{1}{2}-\sqrt{\frac{1}{p}-\frac{3}{4}}, \frac{1}{p}-\frac{1}{2}+\sqrt{\frac{1}{p}-\frac{3}{4}}\right] .
$$


In addition also the restriction concerning $q$ can be weakened. We refer to [9] for details. The authors are convinced that the critical value $p=4 / 3$ and the above "forbidden" interval have nothing to do with the problem itself. To overcome these technical problems one has, probably, to change the method.

Before we start to prove Theorem 1 we indicate how one can simplify the problem. Concerning these reductions we omit details because there are standard in some sense. However, the details can be found in [9].

(1) It will be sufficient to investigate the problem under the following conditions:

$$
\begin{aligned}
& 1+\frac{1}{p}<s \leqslant 2+\frac{1}{p}, \\
& f^{\prime} \in B_{p, q}^{s-1}(\mathbb{R}) .
\end{aligned}
$$

If Theorem 1 is proved under condition (2), then an inductive argument, using the algebra property of $B_{p, q}^{s}(\mathbb{R})$ as well as the continuous embedding $B_{p, q}^{s}(\mathbb{R}) \hookrightarrow B_{p, q}^{s-1}(\mathbb{R})$, yields the general case.

(2) Now we assume that Theorem 1 is proved under the condition (3). Recall that $B_{p, q}^{s}(\mathbb{R})$ is embedded into $C_{b}(\mathbb{R})$. If $f \in B_{p, q}^{s}(\mathbb{R})_{\text {loc }}$ and if $g \in B_{p, q}^{s}(\mathbb{R})$, we consider $\varphi \in \mathcal{D}(\mathbb{R})$ such that $\varphi(x)=1$ on the range of $g$. Then we have $f \circ g=(f \varphi) \circ g$. From this observation the general case follows.

(3) A third reduction consists in assuming that $g$ is a regular function. More precisely, we shall establish the inequality

$$
\|f \circ g\|_{B_{p, q}^{s}(\mathbb{R})} \leqslant c\left\|f^{\prime}\right\|_{B_{p, q}^{s-1}(\mathbb{R})}\left(1+\|g\|_{B_{p, q}^{s}(\mathbb{R})}\right)^{s-(1 / p)}
$$

for any $g \in B_{p, q}^{s}(\mathbb{R})$ such that

$g$ is real analytic.

Then a standard approximation procedure together with the Fatou property of the Besov spaces, see e.g. [7, paragraph $5.2 \&$ Proposition 14], will imply inequality (4) for any $g \in B_{p, q}^{s}(\mathbb{R})$.

\section{Proof of Theorem 1}

We shall prove Theorem 1 by using the assumption

$$
0<\frac{1}{p}<s-[s] .
$$

The proof in case $s-[s] \leqslant 1 / p$ uses the same basic ideas, but is more technical. We refer to [9] for the complete proof, and to [6] for the case $p=\infty$.

We exploit ideas of [7, Theorem 7]. On the one hand we shall use convenient equivalent norms in Besov spaces together with embeddings of Besov spaces into the Wiener space of functions of bounded $p$-variation. On the other hand we shall use monotonicity properties of the regular function $g$.

\subsection{Alternative norms in Besov spaces}

To begin with we deal with integral means of differences. Let

$$
\begin{aligned}
& \Delta_{h} f(x):=f(x+h)-f(x) \quad \forall h, x \in \mathbb{R}, \\
& \omega_{p}(f, h):=\left(\int_{\mathbb{R}}\left|\Delta_{h} f(x)\right|^{p} \mathrm{~d} x\right)^{1 / p}, \quad h \in \mathbb{R} .
\end{aligned}
$$

Assume $m<s<m+1$ for some $m \in \mathbb{N}$. Then the following expression is well known to be an equivalent norm in $B_{p, q}^{s}(\mathbb{R})$ :

$$
\|f\|_{B_{p, q}^{s}(\mathbb{R})}:=\|f\|_{p}+\left(\int_{\mathbb{R}}\left(\frac{\omega_{p}\left(f^{(m)}, h\right)}{|h|^{s-m}}\right)^{q} \frac{\mathrm{d} h}{|h|}\right)^{1 / q} .
$$


We obtain another equivalent norm by replacing, in (7), integration for $h \in \mathbb{R}$ by integration for $|h| \leqslant R$, for a fixed positive number $R$. Indeed the part of the integral for which $|h|>R$ can be easily estimated by the $L_{p}$-norm.

In some cases, an alternative equivalent norm can be obtained as follows. Let

$$
\Omega_{p}(f, t):=\left(\int_{\mathbb{R}} \sup _{|h| \leqslant t}\left|\Delta_{h} f(x)\right|^{p} \mathrm{~d} x\right)^{1 / p}, \quad t>0 .
$$

Then we have the following result, see e.g. [17, Theorem 3.5.3, p. 194]:

Proposition 1. Let $1 / p<s<1$. Then a function $f$ belongs to $B_{p, q}^{s}(\mathbb{R})$ if and only if

$$
\|f\|_{p}+\left(\int_{0}^{\infty}\left(\frac{\Omega_{p}(f, t)}{t^{s}}\right)^{q} \frac{\mathrm{d} t}{t}\right)^{1 / q}<+\infty
$$

Moreover, the above expression generates an equivalent norm on $B_{p, q}^{s}(\mathbb{R})$.

The condition $s>1 / p$ cannot be avoided. Indeed, (8) implies that $f$ is locally bounded, a property which is not shared by all Besov functions for $s<1 / p$.

\subsection{The p-variation of a Besov function}

For a function $g: \mathbb{R} \rightarrow \mathbb{R}$ and any $h \in] 0, \infty]$ we denote by $v_{p}(g, h)$ the supremum of numbers

$$
\left(\sum_{k=1}^{N}\left|g\left(b_{k}\right)-g\left(a_{k}\right)\right|^{p}\right)^{1 / p}
$$

taken over all finite sets \{]$a_{k}, b_{k}[; k=1, \ldots, N\}$ of pairwise disjoint open intervals of length less than $h$. A function $g$ is said to be of bounded p-variation if $v_{p}(g, \infty)<+\infty$. The set of primitives of functions of bounded $p$-variation will be denoted by $B V_{p}^{1}(\mathbb{R})$ and endowed with the semi-norm

$$
\|f\|_{B V_{p}^{1}(\mathbb{R})}:=\inf v_{p}(g, \infty),
$$

where the infimum is taken with respect to all functions $g$ whose primitive is $f$. We refer to $[7,8,18]$ for a discussion of these classes.

Proposition 2. For $1<p<\infty, 1 / p<s<1$, there exists a constant $c=c(s, p, q)>0$ such that

$$
\left(\int_{0}^{\infty}\left(\frac{v_{p}(g, h)}{h^{s-(1 / p)}}\right)^{q} \frac{\mathrm{d} h}{h}\right)^{1 / q} \leqslant c\|g\|_{B_{p, q}^{s}(\mathbb{R})},
$$

for all $g \in B_{p, q}^{s}(\mathbb{R})$.

Proof. By Peetre's embedding theorem, see [13, p. 112] or [7, Theorem 5], there exists $c_{1}=c_{1}(p)>0$ such that

$$
v_{p}(g, h) \leqslant c_{1}\|g\|_{B_{p, 1}^{1 / p}(\mathbb{R})}, \quad \forall h>0, \forall g \in B_{p, 1}^{1 / p}(\mathbb{R}) .
$$

Now we claim that

$$
v_{p}(g, h) \leqslant h^{1-(1 / p)}\|g\|_{W_{p}^{1}(\mathbb{R})}, \forall h>0, \forall g \in W_{p}^{1}(\mathbb{R}) .
$$

Obviously, Hölder's inequality yields

$$
\left|g\left(b_{k}\right)-g\left(a_{k}\right)\right| \leqslant\left(b_{k}-a_{k}\right)^{1-(1 / p)}\left(\int_{a_{k}}^{b_{k}}\left|g^{\prime}(x)\right|^{p} \mathrm{~d} x\right)^{1 / p}, \quad \forall g \in W_{p}^{1}(\mathbb{R}) .
$$


Inequality (11) follows now by taking the $p$-th power and summing up over $k$.

Next we use real interpolation of Banach spaces, cf. e.g. [1] or [15]. Let $\theta \in] 0,1[$ be such that $\theta(1-(1 / p))=$ $s-(1 / p)$. As it is well known,

$$
B_{p, q}^{s}(\mathbb{R})=\left(B_{p, 1}^{1 / p}(\mathbb{R}), W_{p}^{1}(\mathbb{R})\right)_{\theta, q}
$$

with equivalent norms, see $[1,6.4]$ or $[15,2.4]$. For $g \in B_{p, q}^{s}(\mathbb{R})$, we denote by $t \mapsto K(t, g)$ the $K$-functional of $g$ relative to the couple $\left(B_{p, 1}^{1 / p}(\mathbb{R}), W_{p}^{1}(\mathbb{R})\right.$ ) (see [1, paragraph 3.1]). By definition, there exist two families $\left(u_{t}\right)_{t>0}$ and $\left(v_{t}\right)_{t>0}$ of functions such that

$$
g=u_{t}+v_{t} \quad \text { and } \quad\left\|u_{t}\right\|_{B_{p, 1}^{1 / p}(\mathbb{R})}+t\left\|v_{t}\right\|_{W_{p}^{1}(\mathbb{R})} \leqslant 2 K(t, g),
$$

for all $t>0$. By inequalities (10) and (11) we obtain

$$
h^{(1 / p)-s} v_{p}(g, h) \leqslant c_{2} K(t, g)\left(h^{(1 / p)-s}+h^{1-s} t^{-1}\right), \quad \forall h, t>0 .
$$

Taking $t:=h^{1-(1 / p)}$ in the above estimation we deduce

$$
\begin{aligned}
\left(\int_{0}^{\infty}\left(\frac{v_{p}(g, h)}{h^{s-(1 / p)}}\right)^{q} \frac{\mathrm{d} h}{h}\right)^{1 / q} & \leqslant c_{3}\left(\int_{0}^{\infty}\left(\frac{K\left(h^{1-(1 / p)}, g\right)}{h^{s-(1 / p)}}\right)^{q} \frac{\mathrm{d} h}{h}\right)^{1 / q} \\
& =c_{4}\left(\int_{0}^{\infty}\left(\frac{K(t, g)}{t^{\theta}}\right)^{q} \frac{\mathrm{d} t}{t}\right)^{1 / q}
\end{aligned}
$$

which proves (9).

\subsection{The details of the proof}

According to the three reductions mentioned in Section 2 we shall work with the following assumptions:

(i) $1+(1 / p)<s<2$,

(ii) $f^{\prime} \in B_{p, q}^{s-1}(\mathbb{R})$,

(iii) $g$ is a real analytic function in $B_{p, q}^{s}(\mathbb{R})$.

Step 1. Preparations. By definition of the Besov norm, it is enough to estimate

$$
\|f \circ g\|_{p}+\left(\int_{-1}^{1}\left(\frac{\omega_{p}\left((f \circ g)^{\prime}, h\right)}{|h|^{s-1}}\right)^{q} \frac{\mathrm{d} h}{|h|}\right)^{1 / q} .
$$

Since

$$
\|f \circ g-f(0)\|_{p} \leqslant\left\|f^{\prime}\right\|_{\infty}\|g\|_{p}
$$

and

$$
\omega_{p}\left(\left(f^{\prime} \circ g\right) g^{\prime}, h\right) \leqslant\left\|f^{\prime}\right\|_{\infty} \omega_{p}\left(g^{\prime}, h\right)+U(h),
$$

where

$$
U(h):=\left(\int_{\mathbb{R}}\left|\Delta_{h}\left(f^{\prime} \circ g\right)(x)\right|^{p}\left|g^{\prime}(x)\right|^{p} \mathrm{~d} x\right)^{1 / p},
$$

we are reduced to prove that

$$
\left(\int_{-1}^{1}\left(\frac{U(h)}{|h|^{s-1}}\right)^{q} \frac{\mathrm{d} h}{|h|}\right)^{1 / q}
$$


can be estimated by the right-hand side of (4).

Step 2. Without loss of generality we may assume $h>0$. The set of zeros of $g^{\prime}$ is discrete, and its complement in $\mathbb{R}$ is the union of a family $\left(I_{l}\right)_{l}$ of nonempty open disjoint intervals. For any $h>0$ we denote by $I_{l}^{\prime}$ the (possibly empty) set of $x \in I_{l}$ whose distance to the right endpoint of $I_{l}$ is greater than $h$, and we set

$$
I_{l}^{\prime \prime}:=I_{l} \backslash I_{l}^{\prime}, \quad a_{l}:=\sup _{I_{l}}\left|g^{\prime}\right| .
$$

By $g_{l}$ we mean the restriction of $g$ to $I_{l}$, hence a strictly monotone smooth function. If $I_{l}^{\prime} \neq \emptyset$, then we have

$$
\left|g\left(g_{l}^{-1}(y)+h\right)-y\right| \leqslant a_{l} h \quad \text { for } y \in g_{l}\left(I_{l}^{\prime}\right),
$$

where $g_{l}^{-1}$ denotes the inverse function of $g_{l}$.

Substep 2.1. By (12) and by a change of variable we find

$$
\int_{I_{l}^{\prime}}\left|\Delta_{h}\left(f^{\prime} \circ g\right)(x)\right|^{p}\left|g^{\prime}(x)\right|^{p} \mathrm{~d} x \leqslant a_{l}^{p-1} \Omega_{p}^{p}\left(f^{\prime}, a_{l} h\right) .
$$

By the Minkowski inequality w.r.t. $L_{q / p}$, and by Proposition 1, we obtain

$$
\begin{aligned}
\left(\int_{0}^{\infty}\left(\frac{1}{h^{(s-1) p}} \sum_{l} a_{l}^{p-1} \Omega_{p}^{p}\left(f^{\prime}, a_{l} h\right)\right)^{q / p} \frac{\mathrm{d} h}{h}\right)^{1 / q} & \leqslant\left(\sum_{l}\left(\int_{0}^{\infty}\left(\frac{1}{h^{p(s-1)}} a_{l}^{p-1} \Omega_{p}^{p}\left(f^{\prime}, a_{l} h\right)\right)^{q / p} \frac{\mathrm{d} h}{h}\right)^{p / q}\right)^{1 / p} \\
& =\left(\sum_{l} a_{l}^{p-1+p(s-1)}\right)^{1 / p}\left(\int_{0}^{\infty}\left(\frac{\Omega_{p}\left(f^{\prime}, t\right)}{t^{s-1}}\right)^{q} \frac{\mathrm{d} t}{t}\right)^{1 / q} \\
& \leqslant c\left\|f^{\prime}\right\|_{B_{p, q}^{s-1}(\mathbb{R})}\left(\sum_{l}\left(\sup _{I_{l}}\left|g^{\prime}\right|\right)^{s p-1}\right)^{1 / p} .
\end{aligned}
$$

Now we follow [7, proof of Theorem 7]. By definition, the function $g^{\prime}$ vanishes at the endpoints of $I_{l}$. Let $\beta_{l}$ be one of these endpoints. Furthermore, there is at least one point $\xi_{l} \in I_{l}$ such that

$$
\left|g^{\prime}\left(\xi_{l}\right)\right|=\sup _{I_{l}}\left|g^{\prime}\right| .
$$

Hence

$$
\sum_{l} \sup _{I_{l}}\left|g^{\prime}\right|^{s p-1}=\sum_{l}\left|g^{\prime}\left(\xi_{l}\right)-g^{\prime}\left(\beta_{l}\right)\right|^{s p-1} \leqslant v_{s p-1}\left(g^{\prime}, \infty\right)^{s p-1} .
$$

By (13), we conclude that

$$
\left(\int_{0}^{\infty}\left(\frac{1}{h^{(s-1) p}} \sum_{l} \int_{I_{l}^{\prime}}\left|\Delta_{h}\left(f^{\prime} \circ g\right)(x)\right|^{p}\left|g^{\prime}(x)\right|^{p} \mathrm{~d} x\right)^{q / p} \frac{\mathrm{d} h}{h}\right)^{1 / q} \leqslant c\left\|f^{\prime}\right\|_{B_{p, q}^{s-1}(\mathbb{R})}\|g\|_{B V_{s p-1}^{1}}^{s-(1 / p)} .
$$

Substep 2.2. Since the nonempty intervals $I_{l}^{\prime \prime}$ have length equal to $h$, we have

$$
\sum_{l} \int_{I_{l}^{\prime \prime}}\left|\Delta_{h}\left(f^{\prime} \circ g\right)(x)\right|^{p}\left|g^{\prime}(x)\right|^{p} \mathrm{~d} x \leqslant h\left(2\left\|f^{\prime}\right\|_{\infty}\right)^{p} \sum_{l} \sup _{I_{l}^{\prime \prime}}\left|g^{\prime}\right|^{p} .
$$

As above we observe that $g^{\prime}$ vanishes in at least one endpoint of $I_{l}^{\prime \prime}$. Then arguing as in the preceding substep, we have

$$
\sum_{l} \sup _{I_{l}^{\prime \prime}}\left|g^{\prime}\right|^{p} \leqslant c_{1} v_{p}^{p}\left(g^{\prime}, h\right)
$$


By Proposition 2, and by (15), we deduce that

$$
\left(\int_{0}^{\infty}\left(\frac{1}{h^{(s-1) p}} \sum_{l} \int_{I_{l}^{\prime \prime}}\left|\Delta_{h}\left(f^{\prime} \circ g\right)(x)\right|^{p}\left|g^{\prime}(x)\right|^{p} \mathrm{~d} x\right)^{q / p} \frac{\mathrm{d} h}{h}\right)^{1 / q} \leqslant c_{2}\left\|f^{\prime}\right\|_{\infty}\|g\|_{B_{p, q}^{s}(\mathbb{R}) .}
$$

By (14), (16), and by the embeddings

$$
B_{p, q}^{s}(\mathbb{R}) \hookrightarrow B_{s p-1,1}^{1+1 /(s p-1)}(\mathbb{R}) \hookrightarrow B V_{s p-1}^{1}(\mathbb{R}),
$$

where the first one follows by the condition $s-(1 / p)>1$, and the second by (10), we deduce inequality (4). This ends up the partial proof of Theorem 1.

\section{Concluding remarks}

\subsection{Symbolic calculus on Lizorkin-Triebel algebras}

Lizorkin-Triebel spaces represent generalizations of Sobolev spaces to fractional order of smoothness. We refer to $[16,17]$ for the definition and basic properties. Theorem 1 has a counterpart for this scale as well.

Theorem 2. Let us assume that the following two conditions hold:

- $s>1+(1 / p)$,

- $p>4 / 3$ or $1 / p<s-[s]$.

If $f: \mathbb{R} \rightarrow \mathbb{R}$ is a Borel function such that $f(0)=0$, then $f$ operates in $F_{p, q}^{s}(\mathbb{R})$ if and only if $f \in F_{p, q}^{s}(\mathbb{R})_{\text {loc. }}$.

The basic ideas to prove this counterpart are the same as in case of Besov spaces. However, it becomes more technical. As in case of Theorem 1 the given conditions can be weakened. Also Theorem 2 remains valid under the condition (1). In a sense the result is a little bit better for Lizorkin-Triebel spaces, since there is no longer dependence on $q$. Details may be found in [9].

\subsection{Local and periodic results}

Theorems 1 and 2 have counterparts for local spaces, and spaces defined on the circle $S^{1}$. They can be deduced by using appropriate cut-off functions, and the so-called lifting property, see [10] and [4, Proposition 2.4]. To avoid repetition we shall use the generic notation $E_{p}^{s}(\mathbb{R})$ instead of $B_{p, q}^{s}(\mathbb{R})$ and $F_{p, q}^{s}(\mathbb{R})$, respectively. Similarly, $E_{p}^{s}(\mathbb{R})_{\text {loc }}$ replaces $B_{p, q}^{s}(\mathbb{R})_{\text {loc }}$ as well as $F_{p, q}^{s}(\mathbb{R})_{\text {loc }}$.

Theorem 3. Under the same restrictions as either in Theorem 1 (in case of the Besov spaces) or in Theorem 2 (in case of the Lizorkin-Triebel spaces) we have the following:

(i) if $f_{1}$ and $f_{2}$ are functions in $E_{p}^{s}(\mathbb{R})_{\text {loc }}$, then the same is true for $f_{1} \circ f_{2}$;

(ii) if $f_{1}$ and $f_{2}$ are functions in $E_{p}^{s}\left(S^{1}, S^{1}\right)$, then the same is true for $f_{1} \circ f_{2}$.

By definition, a function $f: S^{1} \rightarrow \mathbb{C}$ belongs to $E_{p}^{s}\left(S^{1}\right)$ if the function $x \mapsto f\left(\mathrm{e}^{\mathrm{i} x}\right)$ belongs to $E_{p}^{s}(\mathbb{R})_{\text {loc }}$. We denote by $E_{p}^{s}\left(S^{1}, S^{1}\right)$ the set of functions $f \in E_{p}^{s}\left(S^{1}\right)$ such that $|f(z)|=1$ for all $z \in S^{1}$.

\subsection{Symbolic calculus in the multi-dimensional situation}

The conjecture applies as well to the Banach algebras $E_{p}^{s}\left(\mathbb{R}^{n}\right) \cap L_{\infty}\left(\mathbb{R}^{n}\right)$, see [14, 4.6.4, p. 222]. In 1970 J. Peetre [12] studied the composition of smooth functions $f$ with functions $g \in B_{p, q}^{s}\left(\mathbb{R}^{n}\right) \cap L_{\infty}\left(\mathbb{R}^{n}\right)$. The method he used consisted in a combination of nonlinear interpolation, Gagliardo-Nirenberg inequality, and the multiplication properties 
of Besov spaces. Picking up the same principles, we can prove the following almost optimal superposition theorem for Besov spaces defined on $\mathbb{R}^{n}$, see [5] for more details.

Theorem 4. Let the numbers $s, s^{\prime}, p$ satisfy the conditions $s^{\prime}>s>1$ and $1 / p<s-[s]$. For all function $f$ such that $f(0)=0, f \in B_{p, \infty}^{s^{\prime}}(\mathbb{R})_{\text {loc }}$, and all function $g \in B_{p, q}^{s}\left(\mathbb{R}^{n}\right) \cap L_{\infty}\left(\mathbb{R}^{n}\right)$, the composed function $f \circ g$ belongs to $B_{p, q}^{s}\left(\mathbb{R}^{n}\right)$.

\section{Acknowledgements}

We would like to thank Massimo Lanza de Cristoforis for careful reading of the manuscript and for many valuable discussions during the preparation of this article.

\section{References}

[1] J. Bergh, J. Löfström, Interpolation Spaces, Springer, Berlin, 1976.

[2] G. Bourdaud, Le calcul fonctionnel dans les espaces de Sobolev, Invent. Math. 104 (1991) 435-446.

[3] G. Bourdaud, Fonctions qui opèrent sur les espaces de Besov et de Triebel, Ann. Inst. H. Poincaré Anal. Non Linéaire 10 (1993) $413-422$.

[4] G. Bourdaud, Une propriété de composition dans l'espace $H^{s}$, C. R. Acad. Sci. Paris, Ser. I 340 (2005) 221-224.

[5] G. Bourdaud, Une propriété de composition dans l'espace $H^{s}$ (II), C. R. Acad. Sci. Paris, Ser. I 342 (2006) 243-246.

[6] G. Bourdaud, M. Lanza de Cristoforis, Functional calculus in Hölder-Zygmund spaces, Trans. Amer. Math. Soc. 354 (2002) $4109-4129$.

[7] G. Bourdaud, M. Lanza de Cristoforis, W. Sickel, Superposition operators and functions of bounded p-variation, Rev. Mat. Iberoamer., in press. Prépublication 362 (Fév. 2004), Institut de Mathématiques de Jussieu, Unité Mixte de Recherche 7586, Université Paris VI et Paris VII/CNRS, http://www.institut.math.jussieu.fr.

[8] G. Bourdaud, M. Lanza de Cristoforis, W. Sickel, Superposition operators and functions of bounded p-variation. II, Nonlinear Anal. Ser. A 62 (2005) 483-518.

[9] G. Bourdaud, M. Moussai, W. Sickel, Towards sharp superposition theorems in Besov and Lizorkin-Triebel spaces, submitted for publication.

[10] J. Bourgain, H. Brezis, P. Mironescu, Lifting in Sobolev spaces, J. Anal. Math. 80 (2000) 37-86.

[11] Y. Katznelson, An Introduction to Harmonic Analysis, Dover, New York, 1976.

[12] J. Peetre, Interpolation of Lipschitz operators and metric spaces, Mathematica (Cluj) 12 (1970) 325-334.

[13] J. Peetre, New Thoughts on Besov Spaces, Duke Univ. Math. Ser., vol. I, Duke Univ. Press, Durham, NC, 1976.

[14] T. Runst, W. Sickel, Sobolev Spaces of Fractional Order, Nemytskij Operators, and Nonlinear Partial Differential Equations, De Gruyter, Berlin, 1996.

[15] H. Triebel, Interpolation Theory, Function Spaces, Differential Operators, VEB Deutscher Verlag der Wissenschaften, Berlin, 1978.

[16] H. Triebel, Theory of Function Spaces, Birkhäuser, Basel, 1983.

[17] H. Triebel, Theory of Function Spaces II, Birkhäuser, Basel, 1992.

[18] N. Wiener, The quadratic variation of a function and its Fourier coefficients, J. Math. Phys. 3 (1924) $72-94$. 